\title{
Estudo observacional do efeito de fluazinam sobre a produtividade da cultura da soja - Metanálise
}

\author{
Fernando Luiz Buss Tupich; Marcelo Giovanetti Canteri; Rodrigo Yoiti Tsukahara; \\ André Luis da Silva; Lucas Henrique Fantin
}

\begin{abstract}
Departamento de Agronomia, Universidade Estadual de Londrina, Caixa Postal 6001, CEP 86051-970, Londrina, PR, Brasil
Autora para correspondência: Fernando Luiz Buss Tupich (ftupich@hotmail.com)

Data de chegada: 31/03/2017. Aceito para publicação em: 28/09/2017.
\end{abstract}

$10.1590 / 0100-5405 / 177863$

\section{RESUMO}

Tupich, F.L.B.; Canteri, M.G.; Tsukahara, R.Y.; Silva, A.L.; Fantin, L.H. Estudo observacional do efeito de fluazinam sobre a produtividade da cultura da soja - Metanálise. Summa Phytopathologica, v.44, n.4, p.394-399, 2018.

O fungo Sclerotinia sclerotiorum incide sobre a cultura da soja causando danos em sua produtividade. Entretanto, a quantificação de tais danos ainda demanda estudos, devido à interação com o ambiente em que o desenvolvimento da doença se insere. Assim, o presente estudo tem como objetivo quantificar em estudos observacionais, através de metanálise, a influência que o patógeno exerce sobre a cultura da soja e analisar a viabilidade do controle químico do mesmo com o fungicida fluazinam. Conduziram-se 121 estudos observacionais durante as safras 2011/2012 a 2014/2015 em que cada estudo consistia de uma parcela aplicada e outra sem aplicação do fungicida fluazinam. Agrupandose os estudos por cultivares e safra de cultivo, estabeleceram-se 16 entradas de dados $(\mathrm{K}=16)$ para condução de metanálise em modelos aleatórios.
Observou-se que há diferença entre as respostas das cultivares avaliadas em produtividade em resposta a aplicação do fungicida. Ainda, como estimativa metanalítica, constatou-se que há resposta positiva em 243,44 kg.ha-1 com amplitude de 142,83 a 344,05 kg.ha ${ }^{-1}$. Para a região estudada, no sul do Paraná, a probabilidade de haver qualquer acréscimo de produtividade foi de $88,3 \%$, sendo que para aumento em $60,120,300,600 \mathrm{Kg} \cdot \mathrm{ha}^{-1}$ as mesmas foram 81,5; 72,$7 ; 69,9 ; 39,1$ e $4,1 \%$, respectivamente. Considerando-se R $\$ 70,00$ o custo da saca e R \$160,00.ha ${ }^{-1}$ o custo da aplicação do fungicida, estimou-se em 69,9 $\%$ a probabilidade de retorno financeiro, quando utilizado o mesmo. Assim, a aplicação de fluazinam contribuiu no acréscimo de produtividade de soja frente à incidência de S.scleotiorum.

Palavras-chave: Sclerotinia sclerotiorum, mofo-branco, modelo de efeitos aleatórios, estudos observacionais.

\section{ABSTRACT}

Tupich, F.L.B.; Canteri, M.G.; Tsukahara, R.Y.; Silva, A.L.; Fantin, L.H. Observational study of the effect of fluazinam on culture soybean productivity - Meta-analysis.. Summa Phytopathologica, v.44, n.4, p.394-399, 2018.

The fungus Sclerotinia sclerotiorum affects the soybean crop by causing yield loss. However, quantification of such damage still demands studies due to the interaction with the environment where the disease is inserted. Thus, the present paper has as objective to quantify in observational studies, by meta-analysis, the influence of the pathogen on soybean crop and to analyze the viability of chemical control with the fungicide fluazinam. The conducted observational trials accounted for 121 during the seasons 2011/2012 to 2014/2015, in which each study consisted of a plot with application and another plot without application of the fungicide fluazinam. Grouping the studies according to cultivars and crop season, 16 data entries $(\mathrm{K}=16)$ were established for meta-analysis in random models. There were differences between the cultivars evaluated for yield in response to fungicide application. Moreover, as meta-analytical estimate, there was a positive response at $243.44 \mathrm{~kg}$. ha ${ }^{-1}$, with amplitude from 142.83 to $344.05 \mathrm{~kg} \cdot \mathrm{ha}^{-1}$. For the studied region, in the south of Paraná State, Brazil, the probability for yield increase was $88.3 \%$, and for an increase of $60,120,300$ and $600 \mathrm{~kg} \cdot \mathrm{ha}^{-1}$ the probability was 81.5 ; $72.2 ; 69.9 ; 39.1$ and $4.1 \%$, respectively. Considering R $\$ 70.00$ the cost of soybean bag and $\mathrm{R} \$ 160.00 . \mathrm{ha}^{-1}$ the cost of fungicide application, 69.9 $\%$ was estimated as the probability of financial return when using the fungicide. Thus, fluazinam application contributed to increase soybean yield against $S$. sclerotiorum incidence.

Keywords: Sclerotinia sclerotiorum, white mold, random effects model, observational studies.

Com relação planta-patógeno registrada em 706 hospedeiros, o fungo Sclerotinia sclerotiorum (Lib de Bary) é patógeno causador do mofo-branco da soja, doença de solo, disseminada principalmente por máquinas agrícolas, solo infestado e sementes, tanto pela infecção do tegumento das mesmas com seu micélio, como pela presença de escleródios misturados às sementes na colheita $(1,7)$.

O fungo $S$. sclerotiorum pode colonizar toda parte aérea da planta. As partes infectadas perdem a cor, tornando-se posteriormente pardas, com aparência de podridão mole nos tecidos. Posteriormente, sob condições ambientais favoráveis, desenvolve-se micélio branco e cotonoso, provocando a morte dos tecidos. Nesta fase, ocorre o enovelamento micelial, que originará os escleródios. Estes são inicialmente brancos, tornando-se negros após sua maturação, podendo localizar-se tanto externa, quanto internamente às plantas. Após caírem ao solo, os escleródios são submetidos a variações de temperatura e umidade que, quando favoráveis, estimulam a germinação e desenvolvimento dos apotécios. Os corpos de frutificação produzem ascósporos, esporos que liberados no ar podem causar a infecção das plantas ao depositarem-se nas pétalas senescidas $(1,3,11,13)$. 
A agressividade do patógeno, e consequente os danos à cultura são influenciados por fatores ambientais, determinando diferentes níveis de interação com o hospedeiro, o que dificulta a quantificação do dano proporcionado pelo mofo-branco e análise da viabilidade de seu controle (15).

Meyer et al. (15), estudando o efeito de diferentes fungicidas sobre o controle de mofo-branco em 9 locais durante os anos de 2009 a 2012, constataram redução e produtividade de 242 a 1.083 $\mathrm{kg} \cdot \mathrm{ha}^{-1}$ causado pelo patógeno na cultura da soja. Mesmo em locais onde há alta quantidade de inóculo do fungo, a resposta em produtividade frente a aplicação de fungicida apresentou variação devido principalmente a quantidade de inóculo e do ambiente nos diferentes locais.

Uma vez que há dificuldades na quantificação de danos do mofo-branco, há a oportunidade da aplicação de diferentes técnicas experimentais para colaborar com a melhor estimativa dos danos causados.

Com relação à interpretação e condução de estudos, pode-se classificá-los quanto à forma em que os dados são coletados, em estudos experimentais e observacionais. Enquanto no primeiro caso há a intervenção do pesquisador em controlar fatores que possam influenciar no resultado, no caso de estudos observacionais não há intervenção do pesquisador, somente observa e registra as informações que lhe interessam para a análise em questão (2).

Diferentemente dos estudos experimentais, em estudos observacionais não há a comparação de tratamentos e sim de populações, assim, a análise de estudos observacionais pode ser conduzida segmentando-se dentro da população do estudo, indivíduos que possuem características semelhantes, o que permite com que sejam identificadas causas para o efeito analisado no estudo em questão sob diferentes grupos populacionais (5).

Estudos observacionais são comumente utilizados na área de saúde com o objetivo de determinar a frequência com que um fator está associado à um efeito estudado, fator este, que pode variar de acordo com a população amostrada, conforme estudado por Bastos e Duquia (2), Duarte et al. (6) e Otero et al. (17).

A forma com que estudos observacionais são analisados são as mesmas que estudos experimentais, entretanto, a diferença está na interpretação, uma vez que há dificuldade em se isolar os efeitos que interessam no estudo devido a influência de diversas variáveis devido a heterogeneidade dos dados, os quais são oriundos de diferentes populações (5).

Como opção para análise sistemática de diferentes estudos, há possibilidade de se utilizar metanálise para interpretação dos mesmos. Mais comumente utilizada em revisões de dados publicados, a metanálise considera que a variabilidade de cada estudo proporciona seu nível de contribuição na estimativa global (estimativa metanalítica). Basicamente os estudos são ponderados por modelos, chamados de modelos de efeitos fixos ou aleatórios (23).

A metanálise em modelos fixos supões que todos os estudos são iguais (não há heterogeneidade), e que toda diferença entre eles está associada a erro experimental. Já o modelo de efeitos aleatórios assume que os estudos sofrem diferentes influências de fatores específicos de cada local, havendo heterogeneidade entre os estudos $(4,21,12,16)$.

As causas da heterogeneidade entre estudos são analisadas através de outro modelo metanalítico, o modelo de efeitos mistos. Este assume que há variáveis que explicam a heterogeneidade, chamadas de covariáveis ou variáveis moderadoras. As covariáveis são agrupamentos de características comuns aos estudos, como por exemplo, pode-se agrupar os estudos que possuam a mesma cultivar, tipo de solo, características climáticas ou de epidemiologia de doença, dentre outros $(4,21,12,16)$.

Conduzindo metanálise a partir de revisão sistemática de 43 trabalhos publicados, Tupich (24) estimou em 512,77 kg.ha-1 o decréscimo de produtividade da cultura da soja quando não houve a aplicação do fungicida fluazinam visando manejo de mofo-branco. Ainda, constatou que houve $89,1 \%$ de probabilidade de que haja resposta positiva em produtividade quando utilizado o fungicida, probabilidade decrescente à medida que se estima maior resposta em produtividade $(82,8,69,6,41,7,17,6$ e 4,9\% para ganhos em 120,300, 600, 900 e $1.200 \mathrm{~kg} \cdot \mathrm{ha}^{-1}$ respectivamente).

Assim, devido a heterogeneidade no comportamento, apresentada pelo patógeno $S$. sclerotiorum, o presente estudo tem como objetivo quantificar, através da metanálise, o efeito que o patógeno exerce sobre a cultura da soja e analisar a viabilidade do controle químico do mesmo com o fungicida fluazinam.

Foram conduzidos estudos observacionais durante quatro safras (safra 2011/2012; 2012/2013;2013/2014 e safra 2014/2015), nos estados do Paraná e São Paulo na cultura da soja. Para tal, foram selecionadas 31 propriedades rurais, nas quais selecionaram-se três talhões por propriedade.

Em cada talhão foi alocada uma área observacional, constituída de duas parcelas: a primeira com aplicação do fungicida fluazinam (Frowncide $500 \mathrm{SC}$ ) trabalhado na dose e época de aplicação recomendada pelo fabricante do produto, $1.000 \mathrm{~mL}$ de produto comercial por hectare em duas aplicações (estádio R1 + 7 a 10 dias) e a segunda parcela sem tratamento com fungicida para o controle de Sclerotinia sclerotiorum.

Cada área observacional foi conduzida de acordo com o manejo do local, ou seja, com todos os tratos culturais praticados pelo produtor. Assim, dentre as 124 áreas conduzidas durante as quatro safras, variações em virtude de rotação de culturas influenciaram no número total de áreas consideradas no estudo, totalizando-se 121 áreas observacionais, três áreas foram descartadas devido a impossibilidade de mensuração de produtividade.

Para a estimativa da variabilidade dos dados observados, necessária para condução da metanálise, agruparam-se os dados observacionais por safra e cultivar de soja para condução de análise de variância, totalizando-se então 16 estudos para análise $(K=16)$, relacionados na Tabela 1 .

Em cada área observacional avaliaram-se incidência e severidade de doença e produtividade $\left(\mathrm{kg} \cdot \mathrm{ha}^{-1}\right)$ para o entendimento do nível de intensidade de doença em cada estudo.

Avaliaram-se a incidência e severidade estimadas no estádio fenológico R6 (final do enchimento de grãos) e a produtividade ao final do ciclo da soja.

Avaliaram-se seis pontos de quatro linhas de plantio x 2 metros de comprimento em cada parcela submetendo para avaliação da doença, escala de notas considerando-se 0 - sem presença de mofobranco; 1 - um ou mais ramo lateral infectado por mofo-branco; 2 - ramos laterais e central infectados por mofo-branco e 3-planta morta devido a incidência de mofo-branco.

Posteriormente, calculou-se índice de severidade de doença através da equação proposta por Mckinney (14) (equação 1):

*índice de Mckinney $\frac{(\%)=\Sigma\left(\text { valor da nota } \mathbf{x} n^{\circ} \text { de plantas com essa nota }\right)}{\left(n^{\circ} \text { total de plantas } \mathbf{x} \text { valor máx. da escala de notas }\right)} \times 100$ 
A produtividade foi avaliada colhendo-se seis pontos por parcela, onde cada ponto consistia de quatro linhas de soja por dois metros de comprimento, convertendo-se os dados em Kg.ha' ${ }^{-1}$.

\section{Pré-analise}

A normalidade dos resultados de estudos individuais foram testados a partir do teste de Shapiro-Wilk.

\section{Medidas descritivas}

A variável resposta utilizada, denominada medida de efeito $(D)$, foi obtida a partir da diferença entre a produtividade do tratamento com fungicida e a testemunha sem aplicação em metodologia adaptada de Paul et al. (19).

$$
D=\text { Prod fung }- \text { Prod test (equação 2) }
$$

Onde, "Prod fung" é a média de produtividade do tratamento com aplicação foliar do ingrediente ativo fluazinam e "Prod test" é a média de produtividade da testemunha.

A variabilidade dos dados inseridos na metanálise foi estimada pelo erro padrão, estimado conforme abaixo:

$$
\text { Erro padrão }=\sigma / n(\text { equação } 3)
$$
estudo.

Onde " $\sigma$ " é o desvio padrão e "n" é o número de repetição de cada

Modelo de efeitos aleatórios

Considerando que o conjunto de estudos tem distribuição normal com o efeito médio $(\mu)$ e variância do efeito $\left(\tau^{2}\right)$ representado pela notação $\theta_{\mathrm{i}} \sim \mathrm{N}\left(\mu, \tau^{2}\right)((20),(25))$, o modelo de efeitos aleatórios utiliza como fontes causadoras de efeito a variação dentro e entre os estudos, Podendo ser escrito como:

$$
\mathrm{Yj}=\theta_{\mathrm{M}}+\zeta_{\mathrm{j}}+\varepsilon_{\mathrm{j}} \text { (equação 4) }
$$

Onde, "j" é o estudo, "Yj" é efeito observado em cada estudo, $\varepsilon_{\mathrm{j}}$ é o erro aleatório do estudo " $\mathrm{j}$ “", $\zeta_{\mathrm{j}}$ é o efeito aleatório de cada estudo “j” e $\theta_{\mathrm{M}}$ é a estimativa metanalítica. Para obter a estimativa global e minimizar a variância, calculou-se a média ponderada, em que o peso atribuído a cada entrada foi dado pelo inverso de sua variância, a partir da variância dentro do estudo $\left(\alpha^{2}\right)$ e entre estudos $\left(S i^{2}\right)$. Em que $W i$ é o peso atribuído as entradas dado por.

$$
W i=1 / \alpha^{2}+S i^{2} \text { (equação 5) }
$$

\section{Heterogeneidade}

Para se determinar o impacto da heterogeneidade da estimativa global, foram calculados os índices $H^{2}$ e $I^{2}$ propostos por Higgins \& Thompson (9), os quais indicam o nível de heterogeneidade presente na metanálise.

Uma vez que o agrupamento dos estudos observacionais foi efetuado considerando-se as cultivares presentes no conjunto total de pontos avaliados e ainda quantidade reduzida de estudos agrupados $(\mathrm{K}=16)$, optou-se pela não realização de metanálise em modelos mistos para identificar possíveis covariáveis que atuem na heterogeneidade dos estudos.

\section{Probabilidade influência na Produtividade}

A partir das estimativas obtidas pelo modelo de efeitos aleatórios foi determinado a probabilidade da influência do tratamento com fluazinam sobre a produtividade, seguindo a metodologia proposta por Paul et al. (18) e Madden e Paul (12). Onde a estimativa da probabilidade é dada por:

$$
p_{\vartheta}=\operatorname{Pr}(\zeta \text { new }>\vartheta)=1-\phi(\vartheta-\zeta) / \sigma \quad(\text { equação 6) }
$$

Sendo que, \new indica o efeito do tratamento a ser estimado, $\vartheta$ é a resposta da produtividade a ser estimada, $\sigma$ variância entre os estudos, $\zeta$ estimativa metanalítica e $\phi$ é função da Distribuição Normal. Foram estipulados probabilidade de retorno positivo da produtividade para os níveis 60, 120, 300 e $600 \mathrm{Kg} \cdot \mathrm{ha}^{-1}$, para tratamentos com a aplicação do ingrediente ativo fluazinam em soja sob influência de mofo-branco. Os valores de probabilidade foram multiplicados por 100 e expressos em porcentagem.

\section{Retorno financeiro}

Ainda, seguindo-se a mesma metodologia de probabilidade de incremento de produtividade, estimou-se a probabilidade do tratamento com fluazinam proporcionar retorno financeiro mediante sua utilização, assim como proposto por Salam et al (22). Para fins de estimativa do custo do tratamento com fluazinam, utilizou-se preço de $\mathrm{R} \$ 70,00$ por saca de soja e custo da aplicação do fungicida em $\mathrm{R} \$ 160,00 \cdot \mathrm{ha}^{-1}$, estimando-se assim o custo de aplicação em 2,28 sacas de soja ou 136,8 kg.ha- ${ }^{-1}$.

Assim, estimou-se a probabilidade de resposta positiva em produtividade no nível de 136,8 kg.ha-1 (equação 6).

\section{Metanálise em modelos aleatórios}

A análise da influência da aplicação de fluazinam sobre a cultura da soja baseou-se nos dados apresentados na Tabela 1, que apresenta os dados submetidos à metanálise. Observou-se variação de $-72,83$ a $647,93 \mathrm{~kg} \cdot \mathrm{ha}^{-1} \mathrm{em} D$ (efeito do tratamento) para variação de intensidade de doença variando de 0 a 29,5 \% e 0 a 29,1 \% de incidência e severidade respectivamente.

Uma vez identificada diferenças de respostas quando utilizado o fluazinam na cultura da soja, a metanálise foi efetuada em modelos aleatórios.

Na Figura 1 encontram-se os resultados obtidos para cada cultivar/ safra, bem como a amplitude das estimativas (li e 1s). Quando as amplitudes das cultivares se sobrepõe em valores estimados, assumese que as mesmas não diferiram significativamente entre si. Ainda, quando a amplitude contém o valor "zero", considera-se então que para aquele estudo o tratamento com o fungicida fluazinam não teve efeito significativo sobre a produtividade. Ao final, é apresentada a estimativa metanalítica 243,44 kg.ha ${ }^{-1}$ e sua amplitude $\left(142,83\right.$ a 344,05 kg.ha $\left.{ }^{-1}\right)$.

A maior parte das cultivares diferiram significativamente entre si quanto à resposta em produtividade frente a aplicação de fluazinam. Entretanto, a cultivar BMX Alvo demonstrou efeito não significativo do tratamento com o fungicida, na safra 2014/2015. Tal pode estar associado à variabilidade ( $\sigma$ e $\mathrm{n}^{\mathrm{o}}$ de repetições) do estudo, que influenciou, juntamente com $D$ na estimativa.

Estudos de resistência genética ao patógenos também são recomendados, uma vez que se verificou comportamento diferente entre cultivares, quando há incidência de doença $(8,10)$. Ainda, os diferentes efeitos de tratamento para cada cultivar podem estar associados a diferentes níveis de tolerância ao patógeno, respondendo em menores níveis ao controle químico.

Garcia et al. (8) e Juliatti et al. (10) estudaram a sensibilidade de diferentes cultivares de soja a $S$. sclerotiorum, constatando que há diferenças entre cultivares quanto a agressividade de colonização do patógeno. Nenhum dos autores trabalharam com as cultivares do 
Tabela 1. Medidas descritivas e caracterização do agrupamento dos pontos observacionais visando controle de $S$. sclerotiorum na cultura da soja, em 16 estudos segmentados por cultivares para metanálise, nas safras 2011/2012, 2012/2013, 2013/2014 e 2014/2015.

\begin{tabular}{|c|c|c|c|c|c|c|c|c|c|c|c|}
\hline \multirow{2}{*}{ Safra } & \multirow{2}{*}{ Cultivar } & \multirow{2}{*}{$\begin{array}{c}n^{0} \text { de } \\
\text { repetições }\end{array}$} & \multirow{2}{*}{$\begin{array}{l}\text { Prod } \\
\text { test }^{(1)}\end{array}$} & \multirow{2}{*}{$\begin{array}{c}\text { Prod } \\
\text { fung }{ }^{(2)}\end{array}$} & \multirow{2}{*}{$D^{(3)}$} & \multirow{2}{*}{$\sigma^{(4)}$} & \multirow{2}{*}{$\begin{array}{c}\text { Erro } \\
\text { Padrão }\end{array}$} & \multicolumn{2}{|c|}{ Incidência de Doença } & \multicolumn{2}{|c|}{ Severidade de Doença } \\
\hline & & & & & & & & Testemunha & Fungicida & Testemunha & Fungicida \\
\hline $2011 / 2012$ & NA 5909 & 6 & 4616.79 & 4543.96 & -72.83 & 557.09 & 92.85 & 2.43 & 0.6 & 2.14 & 1.27 \\
\hline $2011 / 2012$ & BMX Turbo & 2 & 4072.3 & 4509.72 & 437.42 & 616.28 & 308.14 & 17.95 & 2.32 & 19.37 & 5.04 \\
\hline $2011 / 2012$ & BMX Potência & 5 & 3633.42 & 3599.17 & -34.25 & 778.41 & 155.68 & 0.13 & 0.15 & 0.51 & 0.63 \\
\hline $2012 / 2013$ & V Top & 3 & 5609.02 & 5871.09 & 262.07 & 445.66 & 148.55 & 0.12 & 0 & 0.18 & 0 \\
\hline $2012 / 2013$ & NK 3363 & 2 & 4405.45 & 4907.97 & 502.52 & 1038.33 & 519.17 & 13.07 & 5.09 & 16.93 & 5.38 \\
\hline $2012 / 2013$ & NA 5909 & 13 & 4275.23 & 4585.27 & 310.04 & 856.38 & 65.88 & 4.19 & 0.74 & 3.13 & 0.58 \\
\hline $2012 / 2013$ & BMX Ativa & 2 & 4039.5 & 4201.63 & 162.13 & 540.77 & 270.39 & 25.8 & 17.18 & 17.26 & 11.18 \\
\hline $2014 / 2015$ & Intacta & 3 & 4952.31 & 5548.84 & 596.53 & 1540.28 & 513.43 & 14.88 & 8.15 & 7.9 & 3.41 \\
\hline $2014 / 2015$ & BRS 284 & 7 & 3415.32 & 3573.36 & 158.04 & 786.74 & 112.39 & 1.93 & 1.82 & 1.21 & 1.05 \\
\hline $2014 / 2015$ & BMX Alvo & 2 & 3221.96 & 3210.36 & -11.6 & 761.34 & 380.67 & 7.73 & 6.85 & 4.22 & 3.24 \\
\hline $2014 / 2015$ & AS3570IPRO & 5 & 3869.92 & 3918.44 & 48.52 & 715.50 & 143.10 & 18.35 & 9.93 & 10.83 & 4.83 \\
\hline
\end{tabular}

Produtividade média da testemunha (área sem aplicação de fluazinam) em kg.ha ${ }^{-1}$. Produtividade média sob tratamento de fluazinam em kg.ha ${ }^{-1}$. Medida de efeito do tratamento em kg.ha- ${ }^{-1}$. Desvio padrão.

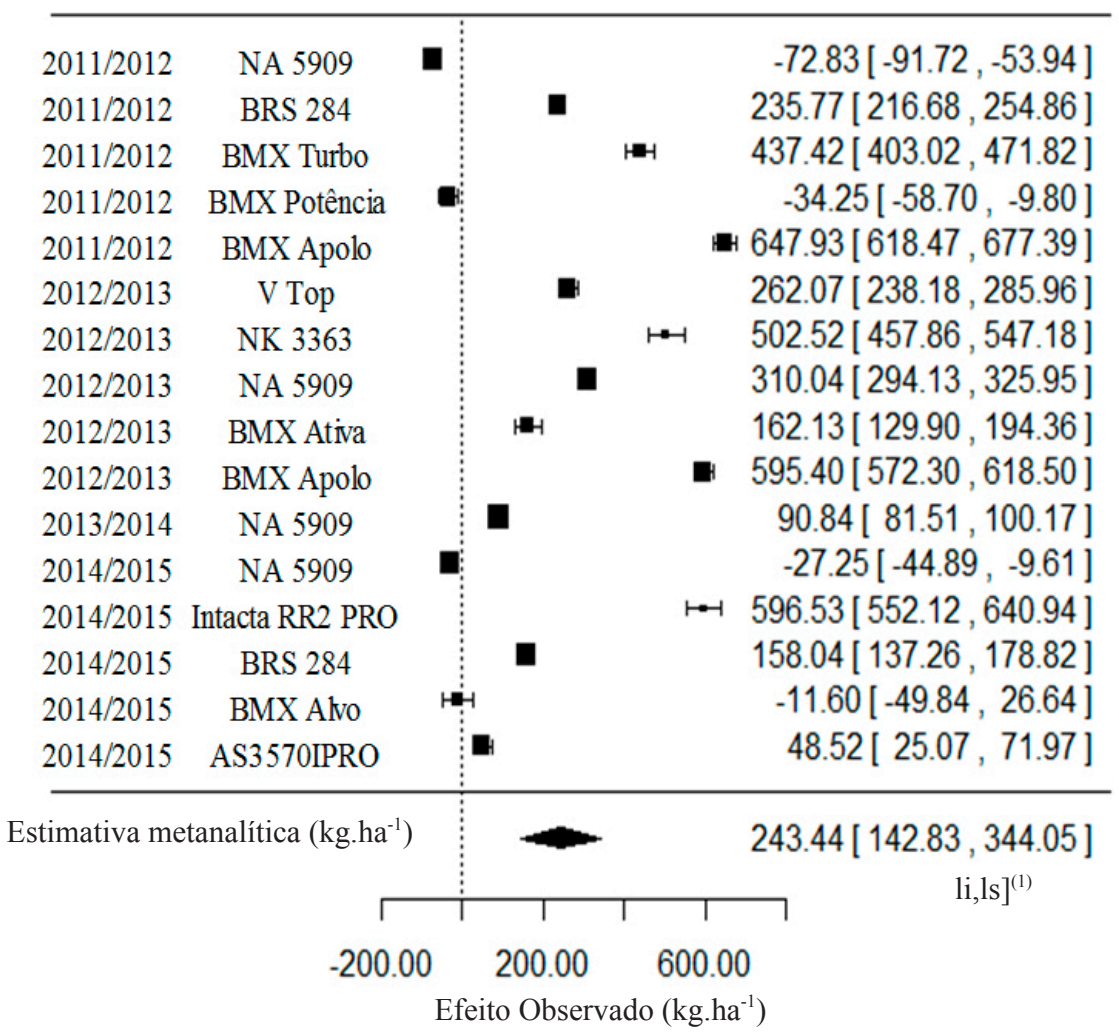

Figura 1. "Forest plot" dos efeitos de tratamento $(D)$ sobre a produtividade (kg.ha-1 ${ }^{-1}$ da cultura da soja e erro padrão de ensaios observacionais conduzidos entre as safras de 2011/2012 e 2014/2015, visando ao controle de S. sclerotiorum com o fungicida fluazinam. 
presente estudo, entretanto a hipótese da interação genética com os resultados obtidos deve ser considerada e pode servir de base para estudos específicos sobre o tema.

Ainda, nota-se que em apenas 3 dos 16 estudos agrupados a estimativa de produtividade significativamente negativa (Prod test $>$ Prod fung), evidenciando-se que nestas situações o tratamento com fluazinam não agregou em maior produtividade, nas quais duas situações ocorreram com a cultivar NA 5909.

Entretanto, o resultado global de todos os agrupamentos é positivo, ou seja, houve benefício do uso do fungicida em produtividade ( $P$ valor $<0,0001$ ) na magnitude de $243,44 \mathrm{~kg} \cdot \mathrm{ha}^{-1}$ e com intervalo de confiança entre 142,83 a $344,05 \mathrm{~kg} \cdot \mathrm{ha}^{-1}$. Uma vez que o intervalo de confiança não contém o valor zero, afirma-se então que o valor estimado é significativo (Tabela 2).

A estimativa constatada no presente estudo é inferior ao observado

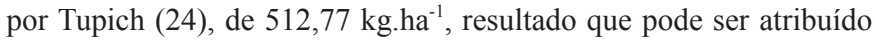
à fonte de dados dos estudos. Utilizou-se no presente estudo dados de campo, com diferentes níveis de intensidade de doença (até 29,5 $\%$ de incidência em plantas), enquanto que para o estudo de Tupich (24) utilizou-se de dados obtidos de publicações, que em sua maioria são estudos conduzidos sob maior pressão de doença (até $64 \%$ de incidência de doença), aumentando assim o dano proporcionado por S. sclerotiorum e consequentemente eleva a resposta em produtividade proporcionada pelo fungicida.

Os dados estimados para os índices $I^{2}$ e $H^{2}$ propostos por Higgins \& Thompson (9), constatam heterogeneidade dos dados, conforme sugerido por Borensten et al. (4). Tal resultado é esperado principalmente devido aos estudos serem de caráter observacional, delineamento que busca maior heterogeneidade dos dados.

Como a metanálise foi conduzida de forma a agrupar os estudos observacionais em cultivares e safras, considerou-se então que a forma de agrupamento já explica parte da heterogeneidade dos dados, considerando-se assim, semelhante à classificação em covariáveis, descartando-se então a subsequente metanálise em modelos mistos, utilizada para explicar a heterogeneidade.

\section{Probabilidade de influência na produtividade}

Uma vez que os dados respeitam distribuição normal, baseando-se na estimativa metanalítica pôde-se estimar a probabilidade de que a aplicação de fluazinam proporcione resposta em diferentes níveis de produtividade (Figura 2).

A aplicação de fluazinam na cultura da soja, tem $88,3 \%$ de probabilidade de que proporcione resposta positiva em produtividade. Em contrapartida, há $11,7 \%$ de probabilidade de que a resposta seja negativa, ou seja, apresente produtividade inferior àquela onde não há o tratamento com o fungicida.

Ainda, à medida que se restringe a resposta positiva de produtividade em maiores níveis (de 60 para 600 kg.ha ${ }^{-1}$ ), há consequente redução de sua respectiva probabilidade (de 81,5 para $4,1 \%$ ). Tal redução é esperada, uma vez que a estimativa da probabilidade pressupõe que os resultados respeitam distribuição normal e assim, quanto maior a distância da estimativa geral, menor é a probabilidade de que a mesma ocorra.

Tabela 2. Estimativa metanalítica, $\mathrm{P}$ valor, Intervalo de confiança e índice $I^{2}$ e $\mathrm{H}^{2}$ para metanálise conduzida em modelos aleatórios para estudos observacionais utilizando o fungicida fluazinam visando ao controle de Sclerotinia sclerotiorum na cultura da soja.

\begin{tabular}{|c|c|c|c|c|c|c|}
\hline \multirow{2}{*}{ Variável } & \multirow{2}{*}{ Estimativa metanalítica } & \multirow{2}{*}{ Pvalor } & \multicolumn{2}{|c|}{ Intervalo de confiança } & \multirow{2}{*}{$\begin{array}{c}I^{2} \\
(\%)\end{array}$} & \multirow{2}{*}{$\boldsymbol{H}^{2}$} \\
\hline & & & Limite inferior & Limite Superior & & \\
\hline Produtividade & 243.44 & $0.0001>$ & 142.83 & 344.05 & 99.72 & 357.62 \\
\hline
\end{tabular}

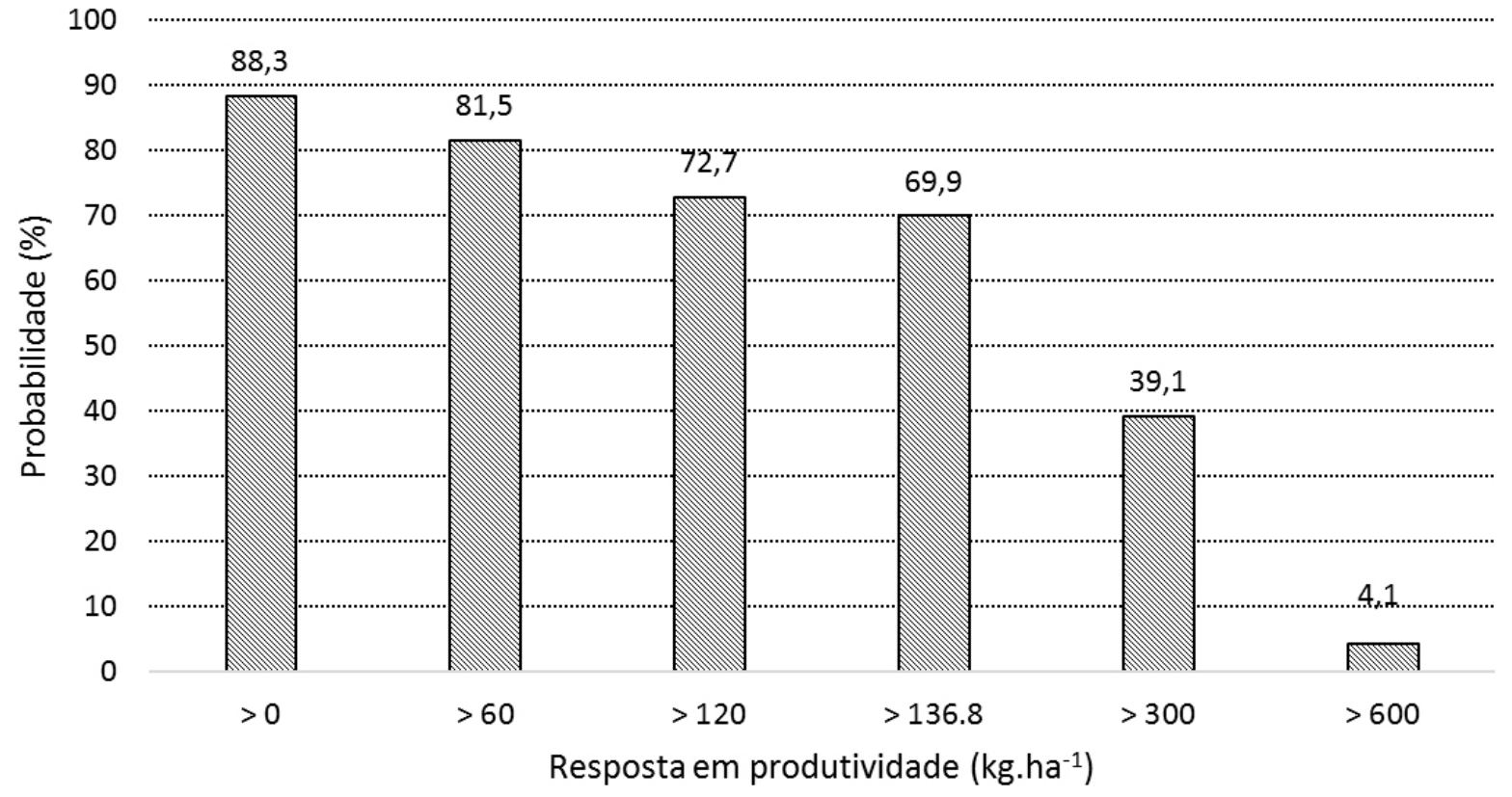

Figura 2. Probabilidade de influência em diferentes níveis de produtividade e de retorno financeiro (\%) do tratamento da cultura da soja com o fungicida fluazinam, visando ao controle de S. sclerotiorum. 
Como variável complementar ao entendimento da viabilidade da aplicação do fungicida e produtividade, considerou-se o custo da aplicação em kg.ha ${ }^{-1}$ como estimativa $\left(136,8 \mathrm{~kg} \cdot \mathrm{ha}^{-1}\right)$. Verificou-se probabilidade de $69,9 \%$ para que haja resposta em produtividade que viesse a equalizar com o custo da aplicação do fungicida.

Embora a estimativa da probabilidade de retorno financeiro ao produtor varie com o preço da soja e do tratamento com o fluazinam, a estimativa serve como ferramenta para decisão do produtor em considerar ou não o manejo químico da doença.

\section{REFERÊNCIAS}

1. Almeida, A.M.R.; Ferreira, L.P..; Yorinori, J.T.; Silva, J.F.V.; Henning, A.A.; Godoy, C.V.; Costamilan, L.M.; Meyer, M.C. Doenças da Soja. In: Kimati, H. Manual de Fitopatologia. $4^{\mathrm{a}}$ ed. São Paulo: Agronômica Ceres, 2005. V.2 Doenças de Plantas cultivadas, p. 569-588.

2. Bastos, J.L.D.; Duquia, R.P. Um dos delineamentos mais empregados em epidemiologia: estudo transversal. Scientia Medica, Porto Alegre, v. 17, n.4, p. 229-232, out/dez 2007.

3. Bianchini .A.; Marigoni, A.C.; Carneiro, S.M.T.P.G. Doenças do Feijoeiro. In: Kimati, H. Manual de Fitopatologia. $4^{\mathrm{a}}$ ed. São Paulo: Agronômica Ceres, 2005. v.2 Doenças de Plantas cultivadas, p. 333-349.

4. Borenstein, M., Hedges, L.V., Higgins, J.P.T., \& Rothstein, H.R. Introduction to Meta-analysis. New Jersey, Wiley and sons. United Kingdom 2009.

5. Cobb, G.W. Introduction to Design and Analysis of Experiments. New York, Springer, 1998, 795p.

6. Duarte, L.; Fujimori, E.; Minagawa,A. T.; Schoeps, F. A.; Montero, R. M. J. M. Aleitamento materno e níveis de hemoglobina em crianças menores de 2 anos em município do estado de São Paulo, Brasil. Revista de Nutrição, Campinas, v.20, n. 2, p. 149-157 2007.

7. Farr, D.F.; Rossman, A.Y. Fungal Databases. US Fungus Collections, ARS, USDA. Disponível em http://nt.ars-grin.gov/fungaldatabases/. Acesso em: 27 out. 2016

8. Garcia, R.A.; Meyer, M.C.; Ávila, K.A.G.B.; Cunha, M.G. Métodos de inoculação de Sclerotinia sclerotiorum para triagem de cultivares de soja resistentes ao Mofo-branco. Pesquisa Agropecuária Brasileira. Brasília: v. 50, n. 8, p.726-729, 2005.

9. Higgins, J.P., \& Thompson, S.G. Quantifyng heterogeneity in a meta-analysis. Statistics in Medicine, London, v. 21, p. 1539-1558, 2002.

10. Juliatti, F.C.; Sagata, E.; Juliatti, B.C.M. Ranqueamento de genótipos de soja com resistência parcial por diferentes métodos de inoculação de Sclerotinia sclerotiorum submetidos à análise de correlação. Bioscience Journal. Uberlândia, v.29, n.3, p $681-689,2013$.

11. Leite, R.M.V.B.C. Doenças do Girassol. In: Kimati, H. Manual de Fitopatologia. $4^{\mathrm{a}}$ ed. São Paulo: Agronômica Ceres, 2005. V.2 Doenças de Plantas cultivadas,p. 385-399, 2005.
12. Madden, L.V.; Paul, P.A. Meta-analysis for Evidence Synthesis in Plant Pathology: An Overview. Phytopathology. v.101, p. 16-30, 2011.

13. Massola, N.S.; Krugner, T.L. Fungos Fitopatogênicos. In: Amorin, L.; Rezende, J.A.M.; Bergamim Filho, A. Manual de Fitopatologia. $4^{a}$ Ed. São Paulo: Agronômica Ceres, 2011. V1 Princípios e Conceitos, p.149-206.

14. Mckinney, H.H. Influence of soil, temperature and moisture on infection of wheat seedlings by Helminthosporium sativum. Journal of Agricultural Research, Washington, v.26, p.195-217, Nov. 1923.

15. Meyer, M.C.; Campos, H.D.; Godoy, C.V.; Utiamada, C.M. Ensaios Cooperativos de controle químico de mofo-branco na cultura da Soja: safras 2009 a 2012. Londrina, EMBRAPA SOJA, Doc 345, 101p, 2014.

16. Ngugi, H.H., Esker, P.D., \& Scherm, H. Meta-Analysis to Determine the Effects of Plant Disease Management Measures: Review and Case Studies on Soybean and Apple. Phytopathology, Saint Paul, v.101, n. 1, p.31-41, 2011

17. Otero, U.; B.; Perez, C. de A.; Szklo, M.; Esteves, G. A.; Pinho, M. M. de; Szklo, A. S.; Turci, S. R. B. Ensaio clínico randomizado: efetividade da abordagem cognitivocomportamental e uso de adesivos transdérmicos de reposição de nicotina, na cessação de fumar, em adultos residentes no Município do Rio de Janeiro, Brasil. Cadernos de Saúde Pública. Rio de Janeiro, v.22, n. 2, p. 439 - 449, 2006

18. Paul, P.A., Lipps, P.E., Hershman, D.E., McMullen, M.P., Draper, M.A., \& Madden, L.V. Efficacy of triazole-based fungicides for fusarium head blight and deoxynivalenol control in wheat: A multivariate meta-analysis. Phytopathology. Saint Paul. v. 98, p. 999-1011. DOI:10. 1094, 2008.

19. Paul, P. A., Madden, L. V., Bradley, C. A., Robertson, A. E., Munkvold, G. P., Shaner, G... Esker, P. Meta-analysis of yield response of hybrid field corn to foliar fungicides in the U.S. Corn Belt. Phytopathology. v,101. p. 1122-1132, 2011

20. R Development Core Team (2011). R: A language and environment for statistical computing. R Foundation for Statistical Computing, Vienna, Austria. ISBN 3-900051-07-0, URL http://www.R-project.org/. Acesso em: 30 out. 2016.

21. Rodrigues, C.L., \& Ziegelman, P.K. Metanálise: Um Guia Prático. Revista HCPA. Porto Alegre. v.30, n. 4, p. 436 - 447, 2010.

22. Salam, K. P., Thomas, G. J., Beard. C., Loughman . R., MacLeod.W.J., \& Salam, M.U. Application of meta-analysis in plant pathology: a case study examining the impact of fungicides on wheat yield loss from the yellow spot—septoria nodorum blotch disease complex in Western Australia. Food Security. London, v 5, p $319-$ 325, 2013.

23. Scherm, H.; Thomas, C.S.; Garrett.K.A.; Olsen, J.M. Meta-Analysis and Other Approaches for Synthesizing Structured and Unstructured Data in Plant Pathology. Annual Review of Phytopathology, Palo Alto, v.52, p.453-476, 2014

24. Tupich, F. L.B.; Canteri. M.G. Metanálise do ganho de produtividade da soja com aplicações de fluazinam para o controle do mofo-branco. 2015. 41f. Dissertação (Mestrado em Agronomia)- UEL - Universidade Estadual de Londrina, Londrina.

25. Viechtbauer, W. Conducing Meta-Analyses in $\mathrm{R}$ with the metaphor Package. Journal of Statistical Software. 36: 3, 1-48, 2010. 